\title{
Role of No Hydro Phacoemulsification Technique in Management of Posterior Polar Cataract
}

\author{
Dr. Gaurav Paranjpe ${ }^{1} \mid$ Dr. Rohini Sangoram ${ }^{2^{*}} \mid$ Dr. Devika Damle
}

\begin{abstract}
${ }^{1}$ Assistant Professor, M.S., Department of Ophthalmology, Krishna Institute of Medical Sciences to Be Deemed University, Karad, 415539;

Maharashtra, India

2 Senior Resident, Department of Ophthalmology,Krishna Institute of Medical Sciences, Karad, 415110 (Maharashtra), India 415539.
\end{abstract}

${ }^{3}$ Junior resident,

Krishna institute of medical sciences, Karad, Maharashtra.

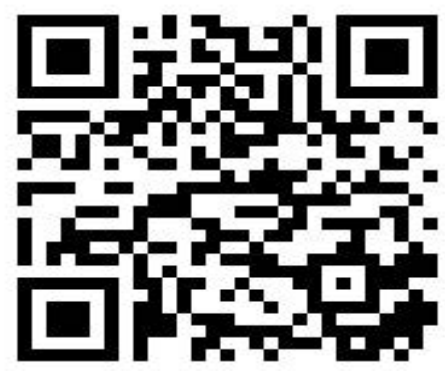

\begin{abstract}
Posterior polar cataracts (PPC) have always been a challenge for cataract surgeons due to their inherently higher propensity for posterior capsule rupture. Over the years, several technical modifications in cataract surgery have been suggested to enhance safety and reduce the percentage of posterior capsule rupture rates in these posterior polar cataracts. This retrospective study tries to present the role and complications of no hydro phacoemulsification technique in management of posterior polar cataract.
\end{abstract}

Aim: To evaluate the role of prehydro phacoemulsification and partial depth phacoemulsification with hydrodelineation in posterior polar cataract (PPC).

Method: Medical records of 100 patients with clinical diagnosis of PPC, who underwent pre hydro phacoemulsification and partial depth phacoemulsification with hydrodelineation, were retrospectively reviewed.

Results : The incidence of posterior capsule rupture (PCR) was $8 \%$ (8/100): 4 cases occurred during epinucleus removal, 3 cases occurred during IOL implantation, and 1 occurred during OVD removal after the implantation of the intraocular lens into the bag. No nucleus piece or lens materials dropped into the vitreous during cataract surgery, and no obvious postoperative complications were found during follow-up. All patients had improved best-corrected visual acuity (BCVA) 1 month postoperatively.

Conclusion: Pre hydro phacoemulsification and partial depth phacoemulsification with hydrodelineation help to reduce the incidence of PCR and achieve satisfactory postoperative outcomes.

Keywords: Posterior polar cataract, Posterior capsular rupture, Hydrodelination, Phacoemulsification, Scleral fixated intraocular lens. 


\section{1 | INTRODUCTION}

$\mathrm{P}$ osterior polar cataract (PPC) is a type of developmental cataract characterized by a white, well-defined, distinctive discoid opacity located on or in front of the central posterior capsule (PC) (1). PPC presents a special challenge to the phaco surgeon due to its high risk of posterior capsule rupture (PCR), vitreous loss, and even nucleus drop during cataract surgery, which can occur because of extreme weakness, pre-existing dehiscence or tight adherence of opacity in the PC (2). In general, cataract surgery, the incidence of PCR is less than $1 \%$ (2), while it is up to $36 \%$ in surgery for PPC (3$5)$. During phacoemulsification, PCR occurs most often during the removal of the epinucleus (5) or the posterior polar opacity (6) for PPC cases. The methods delay the removal of the posterior polar opacity in the epinuclear plate until complete emulsification of the whole nucleus, to minimize the risk for dropping nuclear fragments and losing vitreous (58). In this study, we retrospectively evaluated the results of our case series of pre hydro phacoemulsification and partial depth phacoemulsification with hydrodelineation in PPCs.

\section{2 | METHOD:}

All surgeries were performed by the same surgeon. After topical anesthesia, a $1 \mathrm{~mm}$ side port clear corneal incision was made, followed by injection of a viscoelastic material, a viscous, cohesive gel with $1.5 \%$ sodium hyaluronate to fill the anterior chamber completely. A 2.8-mm, 3-stepped, a clear corneal incision was made superotemporally. Capsulorrhexis was started with the help of 26 gauge cystitome, and continued with a 5.0-5.5 mm continuous curvilinear capsulorrhexis (CCC), taking special care to avoiding viscoelastic escape from the incision.

Partial depth phacoemulsification was done in the centre of nucleus before doing hydro procedure. Hydrodelineation was performed to separate the epinucleus and nucleus at the margin of partial depth phacoemulsification

A Peristaltic system PHACO machine Oertli catarrhex 3 was set to a perimeter lower than normal at power $35 \%$, vacuum $280 \mathrm{mmHg}$, and bottle height $70 \mathrm{~cm}$. The phaco-chop technique was used. After the first division of the nucleus, we rotated the phaco tip towards one-half of the nuclear piece, followed by chopping and emulsifying in situ, avoiding any rotation of the lens pieces. Then for the residual nucleus pieces, we left the integrated posterior epinucleus in situ. The above viscoelastic was injected carefully between PPC and Posterior capsule(PC) from 3 o'clock to 9 o'clock, to lift the epinucleus with the PPC and push down the PC. For large-sized PPC, bi-directional OVD-assisted hydrodissection was performed to release the synechia between PPC and PC. If the PC was judged to have integrated tentatively, routine irrigation and aspiration (I/A) of the posterior epinucleus and cortex was performed by lowering the vacuum at $280 \mathrm{mmHg}$. A foldable IOL was implanted into the capsular bag. Figure 1

After surgery, all the patients were given Milflodex eye drops (Moxifloxacin $0.5 \%+$ Dexamethasone $0.1 \%) 1$ hourly for the first week, which was tapered over the following 4 weeks. All the patients were routinely examined on the first day, first week, first month, and 6 months postoperatively. The data from the first month were evaluated here. And the comparisons of Best Corrected Visual Acuity(BCVA) (logMAR) between 1 month postoperation and preoperation were analyzed with Student's t-test. $P$ value less than 0.05 (two tails) was considered as statistical difference.

Because of the extremely thin or even defective local posterior capsule of PPC, PCR occurs at a high rate during ECCE, phaco surgery, and even femtosecond laser-assisted cataract surgery $(3,6,9,10)$. PCR is inevitable for some cases of PPCs due to pre-existing posterior capsule defects or strong synechia between

Supplementary information The online version of this article (https://doi.org/10.15520/jcmro.v3i10.35 6) contains supplementary material, which is available to authorized users.

Corresponding Author: Dr. Rohini Sangoram SENIOR RESIDENT, DEPARTMENT OF OPHTHALMOLOGY,KRISHNA INSTITUTE OF MEDICAL SCIENCES, KARAD, 415110 (MAHARASHTRA), INDIA 415539.

Email:drrohs199@gmail.com 
ROLE OF NO HYDRO PHACOEMULSIFICATION TECHNIQUE IN MANAGEMENT OF POSTERIOR POLAR CATARACT
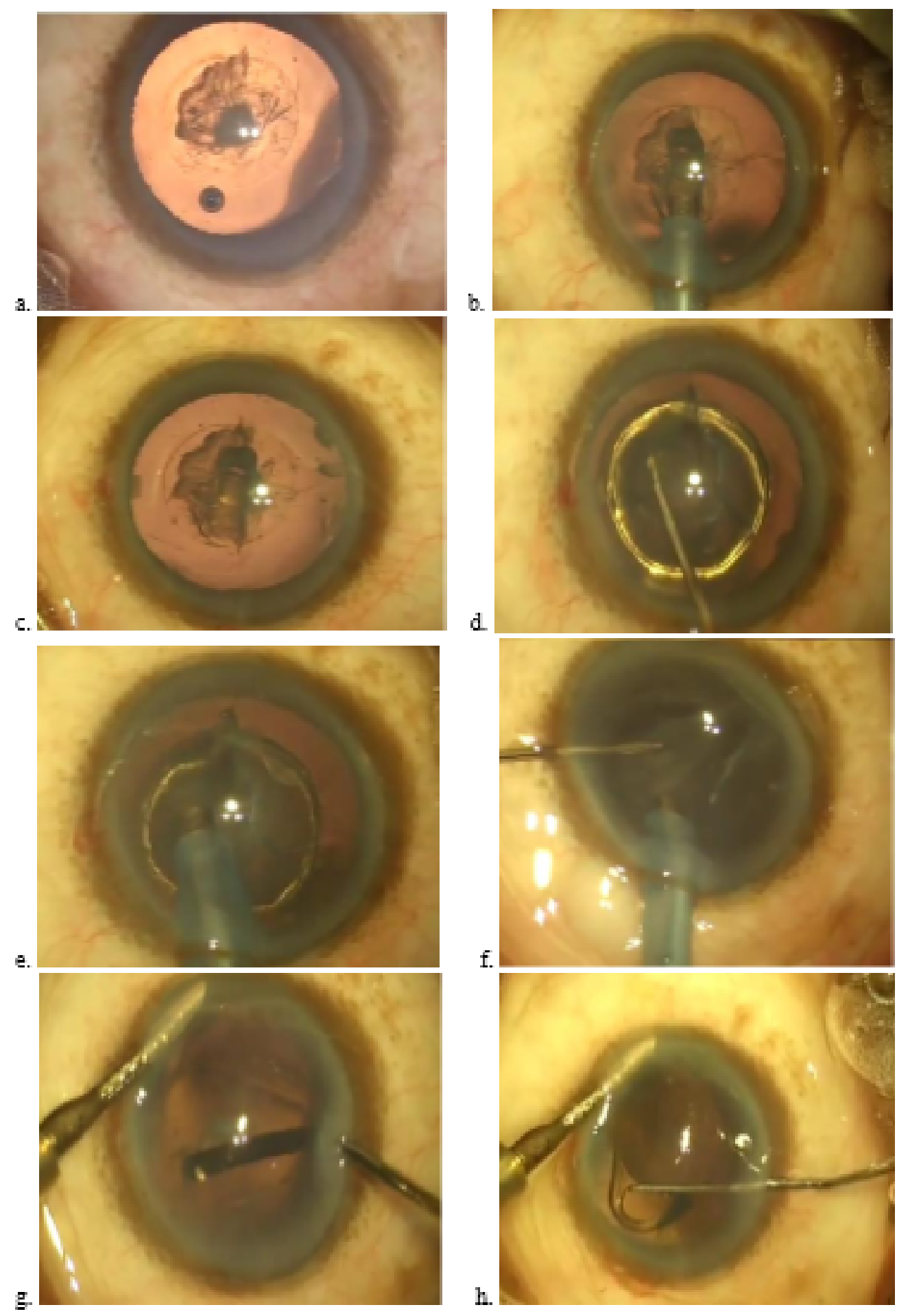

FIGURE 1: Image a. Continuous curvilinearcapsulorrhexis Image b \& c. Partial thicknessphacoemulsification Image d. Hydrodelineation Image

e \& f. Phacoemulsificationof nuclear fragments Image

g. Irrigation aspiration of cortical fibres Image h. IOL implantation 
the posterior opacity and posterior capsule. Special care should be taken at all steps of cataract surgery for such cases.

\section{3 | DISCUSSION:}

In our study, all procedures started with a side port incision followed by injecting viscoelastic into the anterior chamber. We believe that the possibility of anterior chamber collapse might be lower with this approach than starting from the main incision, so this approach might be helpful for avoiding the rupture of a weak PC. Overloading viscoelastic injection should be avoided. For capsulorrhexis, we used 26 gauge cystitome. During the whole maneuver, it was important not to exert pressure on the incision; otherwise, the viscoelastic might overflow and the anterior chamber prolapse. a 5-5.5-mm capsulorrhexis was performed. In our opinion, a too-large capsulorrhexis is not suggested; although it can cause minimal turbulence in the capsular bag during phacoemulsification, if PCR occurs, not enough anterior capsule rim will exist to support the IOL in the sulcus (11). Most previous studies suggested a 5.0- to 5.5-mm circular curvilinear capsulorrhexis. Vasavada et al (5) preferred $4.5 \mathrm{~mm}$ and Pong and Lai et al (12) suggested a 5.5- to $6.0-\mathrm{mm}$ CCC for a hard nucleus. Singh et al (11) described an oval capsulorhexis technique with all grades of nuclear sclerosis for PPC with preexisting PCR, with good results. In our cases, it was not difficult to perform the hydro process and phaco chop with a 5.5-mm-diameter anterior capsule opening, even for a moderate-hardness nucleus. Routine hydrodissection may cause a sudden fluid wave in the weak or defective PC of such cases, which should be avoided right after capsulorrhexis. Many surgeons suggest only hydrodelineation at this stage for PPC $(7,9,13,14)$. We performed conventional hydrodelineation with the cannula penetrating into the lens. As the fluid was injected from outside to inside, usually a golden ring was noticed, which indicated a successful hydrodelineation. A layer of epinucleus was in front of the PC, and the posterior polar opacity was left in situ. Then, phacoemulsification with lower vacuum and lower bottle height was started.
We selected the phaco chop technique in this case series. As the nucleus was divided into pieces, the nuclear material was emulsified in situ, without rotating with the epinuclear plane in front of PC, all of which might exert minimal stress on the capsular bag, especially the weak PC. During the process, it was important not to exert stress on the capsular bag and not to rotate the lens substance until all the nucleus was emulsified and aspirated. Another important issue was to keep the anterior chamber stable. Some viscoelastic could be injected through the side port before withdrawing the phaco tip.

For epinuclear removal a medical sodium hyaluronate gel was used as more OVD was injected, the PC was noticed to be pushed down and the posterior opacity with the epinucleus flowed up, which was followed by the I/A tip instead of the phaco tip to remove the epinucleus gradually, and the residual cortex as well. Posterior capsule polishing should be avoided, even the PC is intact with some opacity, because of the weak nature of such PCs $(5,6,13)$. PCR in 4 cases was noticed during this stage, and some Viscoat was injected between the PCR plane and epinucleus. The epinucleus was then removed by manual dry aspiration with the Simcoe cannula when there was no or minimal vitreous was left out. Otherwise, anterior vitrectomy with a high cutting rate and low vacuum and bottle height was performed until the anterior chamber was free of vitreous. Foldable IOL was then inserted into the bag for the intact PC in cases of PCR.

Compared with other reports of different surgical techniques for PPC, the PCR ratio in our case series is $8 \%$ among which, 4 were found before IOL implantation and in two cases PCR occurred during IOL implantation, and in 4 cases Scleral Fixated Intraocular lens(SFIOL) were put, and other 2 cases of PCR occurred during the removal of OVD in the anterior chamber. Out of total 8 PCR cases, in 3 cases we noted iris prolapsed as intra operative complication but this did not affect final visual outcome.

We observed two cases of PCR when the anterior chamber collapsed after removing all the OVD. In these cases, the PC was intact after the one-piece acrylic IOL was inserted into the bag, and then, routine I/A of viscoelastic material was performed 
normally. After withdrawal of the I/A tip, the anterior chamber was shallowed, and then PCR was found. We believe that this kind of PC is fragile by nature and cannot endure pressure from the posterior segment and IOL as the anterior chamber disappears (9). In post operative complications, raised intraocular pressure was noted in six cases and managed accordingly without affecting the final visual outcome.

Only two eyes did not reach satisfactory postoperative visual acuity, due to macular degeneration with uneventful cataract surgery. All other eyes had improved BCVA, whether PCR existed or not. With our technique of pre hydro phacoemulsification and partial depth phacoemulsification with hydrodelineation, the epinucleus with posterior opacity was easy to remove, and it was safe to handle the lens materials, even those with pre-existing PCR or PCR that occurred during this stage, since the chance of dropping the nucleus was minimal and leakage of vitreous was effectively pushed back.

\section{4 | CONCLUSION:}

In conclusion, PCR is sometimes inevitable in cases of PPC. We adopted different techniques to minimize the damages to the affected eyes. The goals of the surgery are to remove the PPC safely and to keep the integrity of the PC or reduce the chance of dropping lens materials and the loss of vitreous. Pre hydro phacoemulsification and partial depth phacoemulsification with hydrodelineation was an effective treatment for PPCs.

ETHICAL APPROVAL : All procedures performed on human participants were in agreement with ethical standards of the Institutional and/or National Ethics Committee.

SOURCE OF FUNDING : In this project, the cost of investigations of the studyparticipants was born by the institute research fundings.

CONFLICT OF INTEREST : None

\section{REFERENCES}

1. Vasavada AR, Raj SM, Vasavada V, Shrivastav S. Surgical approaches to posterior polar cataract: a review. Eye (Lond). 2012;26(6):761-70.

2. Osher RH, Cionni RJ. The torn posterior capsule: its intraoperative behavior, surgical management, and long-term consequences. J Cataract Refract Surg. 1990;16(4):490-4.

3. Das S, Khanna R, Mohiuddin SM, Ramamurthy B. Surgical and visual outcomes for posterior polar cataract. $\mathrm{Br} \mathrm{J}$ Ophthalmol. 2008;92(11):1476-8.

4. Siatiri H, Moghimi S. Posterior polar cataract: minimizing risk of posterior capsule rupture. Eye (Lond). 2006;20(7):814-6.

5. Vasavada A, Singh R. Phacoemulsification in eyes with posterior polar cataract. J Cataract Refract Surg. 1999;25(2):238-45.

6. Osher RH, Yu BC, Koch DD. Posterior polar cataracts: a predisposition to intraoperative posterior capsular rupture. J Cataract Refract Surg. 1990;16(2):157-62.

7. Allen D, Wood C. Minimizing risk to the capsule during surgery for posterior polar cataract. J Cataract Refract Surg. 2002;28(5):742-4.

8. Fine IH, Packer M, Hoffman RS Management of posterior polar cataract. J Cataract Refract Surg. 2003;29(1):16-9.

9. Consultation section. Cataract surgical problem. J Cataract Refract Surg. 1997;23(6):819-24.

10. Alder BD, Donaldson KE. Comparison of 2 techniques for managing posterior polar cataracts: traditional phacoemulsification versus femtosecond laser-assisted cataract surgery. J Cataract Refract Surg. 2014;40(12):2148-51. 
11. Singh K, Mittal V, Kaur H. Oval capsulorhexis for phacoemulsification in posterior polar cataract with preexisting posterior capsule rupture. J Cataract Refract Surg. 2011;37(7):1183-8.

12. Pong JC, Lai JS. Managing the hard posterior polar cataract. J Cataract Refract Surg. 2008;34(4):530. author reply 530-531

13. Hayashi K, Hayashi H, Nakao F, Hayashi F. Outcomes of surgery for posterior polar cataract. J Cataract Refract Surg. 2003;29(1):45-9.

14. Lee MW, Lee YC. Phacoemulsification of posterior polar cataracts--a surgical challenge. Br J Ophthalmol. 2003;87(11):14267.
How to cite this article: Paranjpe, D. G., Sangoram, D. R., \& Damle, D. D. (2020). Role of No Hydro Phacoemulsification Technique in Management of Posterior Polar Cataract. Journal of Current Medical Research and Opinion, 3(10), 707-711. https://doi.org/10.15520/jcmro.v3i10.356 